\title{
A Finite Volume Approach to Compressible Large Eddy Simulations
}

\author{
B.J.GEURTS, J.G.M. KUERTEN, A.W. VREMAN, V. THEOFILIS and \\ P.J. ZANDBERGEN \\ Department of Applied Mathematics, University of Twente, P.O. Box 217 \\ 7500 AE Enschede, The Netherlands
}

\begin{abstract}
We present Large Eddy Simulations of compressible turbulent flow within a finite volume approach with central spatial differencing. Different spatial discretisations yield quite different predictions of fluctuating flow properties although mean and integral flow properties appeared less sensitive. In the case of homogeneous, isotropic, decaying turbulence the Kolmogorov $-(5 / 3)$ law is reproduced if the convective and viscous fluxes are treated with Simpson's cell vertex method. For the temporal flat plate exponentially growing unstable modes are recovered in the linear regime. Moreover, a scheme in which the viscous fluxes are treated with a 'vertex based' method is required in order to properly capture the saturation of growing fluctuations, due to nonlinear interactions.
\end{abstract}

Key words: finite volume - compressible LES - linear instability

\section{Introduction}

In Large Eddy Simulations (LES) small scale turbulent structures, occurring at high Reynolds numbers, are modeled and the large scale structures are treated directly and time accurately. In most LES-studies a spectral method is introduced for the spatial discretisation (see e.g. ref. [1]). Problems may arise in this approach, however, when treating flows in nonstandard geometries and when shocks are present. In this paper we study the applicability of a finite volume approach to direct- and large eddy simulations. This approach is only second order accurate on smooth grids but has the benefit of being more easily adaptable to complex geometries. Results will be presented for two different applications: a LES-study of compressible, homogeneous, isotropic, decaying turbulence and a temporal direct simulation of instabilities in a compressible flat plate flow. In the first application the Kolmogorov - (5/3) law for the inertial range in the energy spectrum is recovered, even on coarse grids [2]. Moreover, growth rates of unstable modes in a $2 \mathrm{D}$ temporal simulation of compressible flow over a flat plate were obtained in close agreement with results from linear stability theory [3]. Viscous instabilities in wall bounded shear layers at low Mach numbers can be simulated accurately with a finite volume method. A study of inviscid instabilities of the 'tanh-profile' (compressible mixing layer) showed good agreement with linear stability theory as well. It will be shown, however, that different spatial discretisations within a finite volume approach result in quite different predictions of fluctuating flow properties. 
In section 2 we briefly sketch the numerical method. A discussion of homogeneous turbulence is given in section 3 and results for the growth rates of unstable modes in the 2D temporal treatment of a flat plate are presented in section 4 . Some concluding remarks are collected in section 5 .

\section{Formulation of the Numerical Method}

The formulation of the finite volume approach is based on the integral formulation of the Navier-Stokes equations. Integrating these equations over a 'control volume' $\Omega_{\mathrm{i}, \mathrm{j}, \mathrm{k}}$ around a grid point $\mathbf{x}_{\mathrm{i}, \mathrm{j}, \mathrm{k}}$ yields

$$
d_{\mathrm{t}} f_{\mathrm{i}, \mathrm{j}, \mathrm{k}}+\left|\Omega_{\mathrm{i}, \mathrm{j}, \mathrm{k}}\right|^{-1}\left[\int_{\Omega_{\mathrm{i}, \mathrm{j}, \mathrm{k}}} \partial_{l} g_{l} d \mathbf{x}-\int_{\Omega_{\mathrm{i}, \mathrm{j}, \mathrm{k}}} \partial_{l} h_{l} d \mathbf{x}\right] \approx 0
$$

where $f$ denotes the state vector, $f=[\rho, \rho \mathbf{u}, e], g_{l}$ is the convective flux vector in the $x_{l}$-direction and $h_{l}$ the viscous flux vector. Moreover, $\left|\Omega_{\mathrm{i}, \mathrm{j}, \mathrm{k}}\right|$ is the volume of $\Omega_{\mathrm{i}, \mathrm{j}, \mathrm{k}}$ and $d_{\mathrm{t}}, \partial_{l}$ denote derivatives with respect to time and $x_{l}$ respectively. Hence, it is required to obtain accurate approximations of the averages of the convective and viscous fluxes over $\Omega_{\mathrm{i}, \mathrm{j}, \mathrm{k}}$. On an orthogonal grid one may write for the partial derivatives of e.g. the convective fluxes with respect to $x_{1}$ :

$$
\begin{aligned}
\left|\Omega_{\mathrm{i}, \mathrm{j}, \mathrm{k}}\right|^{-1} \int_{\Omega_{\mathrm{i}, \mathrm{j}, \mathrm{k}}} \partial_{1} g_{1} d \mathbf{x} & \approx\left(x_{\mathrm{i}+1}^{(1)}-x_{\mathrm{i}-1}^{(1)}\right)^{-1} \sum_{\alpha=-1}^{1} \sum_{\beta=-1}^{1} w_{\alpha, 2} w_{\beta, 3} \\
& \times\left\{g_{\mathrm{i}+1, \mathrm{j}+\alpha, \mathbf{k}+\beta}^{(1)}-g_{\mathrm{i}-1, \mathrm{j}+\alpha, \mathbf{k}+\beta}^{(1)}\right\}
\end{aligned}
$$

The integration weights $w_{\alpha, l}$ control the contribution of the corresponding point to the integral, when integrating over $x_{l}$. Typical choices for integration over three points are Simpson's rule and the trapezoidal rule. Different integration rules can be adopted for the convective and viscous fluxes. A four stage Runge-Kutta scheme is used for the time-integration of the discretised equations. The convective fluxes in the point $\mathbf{x}_{\mathrm{i}, \mathrm{j}, \mathrm{k}}$ are built from the state vectors at points with $i \pm 1$, whereas the viscous fluxes, involving second order partial derivatives, imply knowledge from points at $i \pm 2$ and at $i$, in the above formulation. Hence, the two types of fluxes are built from disjunct grids. This resulted in numerical instabilities in the flat plate flow. By treating the viscous fluxes with a 'vertex based' method this 'convectiveviscous decoupling' can be removed. In this method the control volume for the viscous fluxes is defined by connecting the midpoints of all the cells containing $\mathrm{x}_{\mathrm{i}, \mathrm{j}, \mathrm{k}}$. Partial derivatives appearing within the viscous flux vector (e.g. $\partial_{\mathrm{i}} u_{\mathrm{j}}, \partial_{\mathrm{i}} T$ ) are calculated directly in these midpoints and the trapezoidal rule is adopted for integration over the cell faces. It will be shown that this treatment of the viscous fluxes is required in order to correctly represent both the initial exponential growth of unstable modes in the flat plate case, as well as the saturation due to nonlinear interactions in the long time regime. 


\section{Compressible, Homogeneous, Isotropic Turbulence}

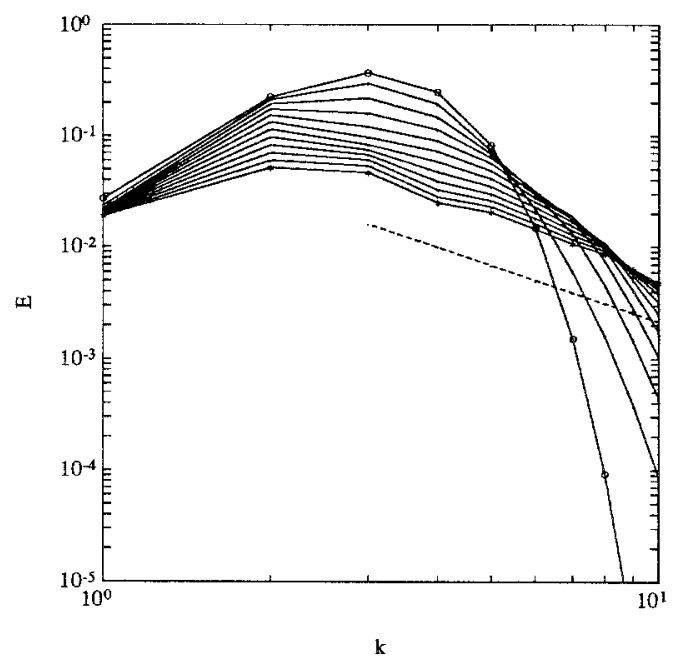

Fig. 1. The evolution of the energy spectrum $E$ obtained with the Simpson cell vertex method, at $M=0.05$ and a Taylor Reynolds number of $40 . \mathrm{A}(21)^{3}$ grid was used. The time interval between two successive curves is 0.06 ; ' 0 ': initial spectrum; ' $*$ ' spectrum at $t=0.6$; dashed line is Kolmogorov's $-(5 / 3)$ law. We used $C_{s}=0.2$ and set the 'filter width' $\Delta$ equal to the grid spacing.

We consider LES of compressible, homogeneous, isotropic, decaying turbulence in a rectangular box in three spatial dimensions. An analysis of the energy spectra obtained from the simulations shows that an agreement with the Kolmogorov law for the inertial range is found only when an appropriate spatial discretisation method is used. This agreement is obtained both for a low (0.05) and a moderate (0.6) Mach number [2]. Smagorinsky's subgrid model is employed to represent the small scales; in this model the eddy viscosity $\left(\mu_{\mathrm{t}}\right)$ is given by

$$
\mu_{\mathrm{t}}=\rho\left(C_{\mathrm{s}} \Delta\right)^{2}\left(S_{\mathrm{ij}} \partial_{\mathrm{i}} u_{\mathrm{j}}\right)^{1 / 2}
$$

where $C_{\mathrm{s}}$ is Smagorinsky's constant and $\Delta$ the filter width. The 'strain rate tensor' $S_{\mathrm{ij}}$ incorporates the divergence of the velocity, and hence this model accounts for some compressibility effects, which become more important at higher Mach numbers. An eddy diffusivity was introduced to model the turbulent heat flow [4].

As was sketched in the previous section, there is no restriction on the assignment of the integration weights $w_{\alpha, \mathrm{n}}$ for treating the spatial discretisation of the fluxes. Different choices can be introduced, which may have widely different effects on the Fourier modes contained in the instantaneous state, and result in a quite different evolution in time. The generation of small scales due to the nonlinear convective fluxes should be in balance with the dissipation of these scales due to the viscous fluxes. In figure 1 we show the energy spectrum in which the convective and 
viscous fluxes were treated on the same control volume and Simpson's rule was used for the integration of the flux vectors over the cell faces. A clear agreement with the Kolmogorov law for the inertial range can be seen. The importance of a balance between the generation and the dissipation of small scales in the discrete equations was observed when using different integration rules. If the convective fluxes are treated with the trapezoidal rule and the viscous fluxes with a 'vertex based' central difference scheme, the dissipation of the small scales was found to be too large, resulting in a far too steep tail in the spectrum. Adopting the midpoint rule for the convective fluxes, shows a far too strong generation of small scales and an accumulation of energy in the tail of the energy spectrum. These observations show that the prediction of fluctuating flow properties depends sensitively on the method used for the spatial discretisation (see ref. [2] for a more detailed discussion).

\section{Temporal Flat Plate}

In this section we focus attention on a 2D temporal treatment of compressible flow over a flat plate. We use periodic boundary conditions in the streamwise direction and adiabatic wall conditions. At the free-stream boundary we use first order extrapolation of Riemann invariants. A forcing field in the streamwise direction is introduced to prevent the flow to develop into an error-function profile (see e.g. refs. $[5,6])$. We use the similarity solution to the compressible boundary layer equations to specify this forcing. A 'white-noise' perturbation is superimposed on this mean velocity profile at $t=0$.

The exponential growth of unstable modes in this flow is considered in order to validate the numerical method. If the perturbations around the mean velocity profile are small, the evolution of the flow should closely follow the predictions obtained from linear stability theory [3]. A comparison between the real $\left(\omega_{\mathrm{r}}\right)$ and imaginary $\left(\omega_{\mathrm{i}}\right)$ parts of the eigenvalues and the corresponding eigenfunctions as obtained from solving the generalised eigenvalue problem, and the results obtained from the simulations was made. In figure 2 we present the evolution of the fluctuating kinetic energy $(E)$ obtained with 'vertex based' discretisation methods, using $R e=1000, M=0.5$ and a computational box of length $L_{1}=31$ scaled on the displacement thickness [6]. Initially many individual eigenmodes are excited which are all stable except for one. Hence, the evolution of $E$ initially shows a decrease, due to the fact that most modes contained in the initial state decay. After this transient only the most amplified mode remains and a linear growth is observed followed by a saturation due to nonlinear interactions. A strong dependence on the spatial discretisation method can be observed although qualitatively the simulations agree. The theoretical predictions $\left(\omega_{\mathrm{r}} \approx 7.12 \cdot 10^{-2}, \omega_{\mathrm{i}} \approx 1.67 \cdot 10^{-3}\right)$ are closely approximated (error less than 0.5 percent) by a scheme in which the trapezoidal rule is used for the convective fluxes on a stretched grid with 64 points in the normal direction. All other methods show large errors on this grid. Moreover, spatial discretisations containing the 'convective-viscous' decoupling yield 


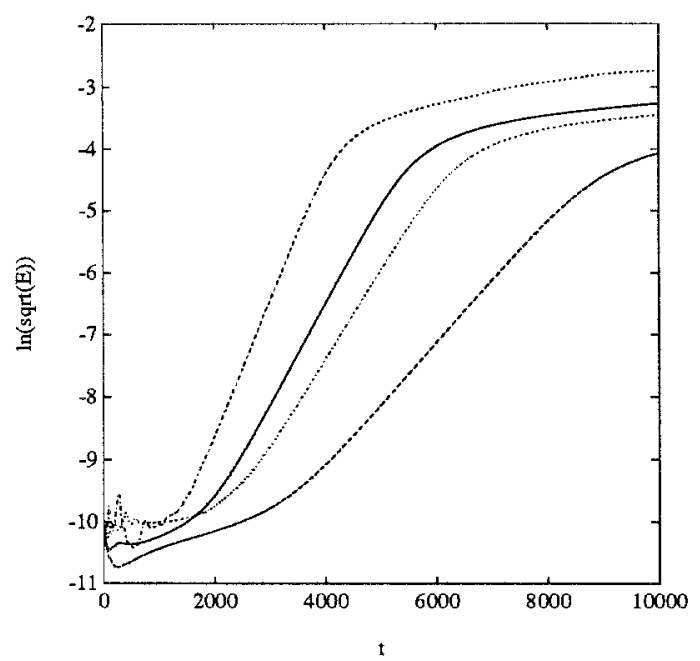

Fig. 2. Simulated evolution of the fluctuating kinetic energy $E$ using different spatial discretisations. A 'vertex based' method for the viscous fluxes is used. The treatment of the convective fluxes was varied; full curve: composite trapezoidal rule, dotted curve: Simpson rule, chain-dotted curve: coarse trapezoidal rule, dashed curve: midpoint rule.

numerical instabilities in the long time regime.

\section{Concluding Remarks}

We have shown that finite volume approaches can be succesfully applied to both DNS and LES of compressible turbulent flow. The fluctuations in several flow properties show a strong influence of the specific scheme which was used for the spatial discretisation. In case of growing unstable modes, a vertex based treatment of the viscous fluxes is needed in order to prevent the 'convective-viscous decoupling' from giving rise to numerical instabilities. In case of homogeneous decaying turbulence in a homogeneous box, this decoupling was found necessary in order to correctly predict the Kolmogorov $-(5 / 3)$ law at intermediate times.

\section{References}

1. Erlebacher, G. Hussaini, M.Y. Kreiss, H.O. and Sarkar,S.: 1990,Int.J. on Theoret.Comput Fluid Dynamics 2, 73 .

2. Vreman, A.W. Geurts, B.J. Kuerten, J.G.M. and Zandbergen, P.J.: 1992, Int.J. Num.Methods in Fluids 15, 799

3. Mack, L.M.: 1984, AGARD-report 709, p5-1.

4. Leith, C.E.: 1990, Physics of Fluids A 2, 297.

5. Spalart, P.R. and Yang, K-S: 1987,JFM 178, 345.

6. Normand, X. and Lesieur, M.: 1992, Theoret. Comput. Fluid Dynamics 3, 231. 